\title{
Redes bayesianas para predecir el estilo de aprendizaje de estudiantes en entornos virtuales
}

\section{Bayesian networks to predict the learning style of student in virtual environments}

\author{
Lissette Geoconda López-Faican ${ }^{1}$, Luis Antonio Chamba-Eras² \\ ${ }^{1}$ Universidad Nacional de Loja (UNL), Loja, Ecuador \\ ${ }^{2}$ Universidad Internacional del Ecuador (UIE), Quito, Ecuador
}

Autor para correspondência/Corresponding author: Luis Antonio Chamba-Eras [ luchambaer@internacional.edu.ec ]

Recebido/Submitted: 30 Out. 2014

Aceito/Approved: 26 Nov. 2014

cC)(1) Copyright ( 2014 López-Faican \& Chamba-Eras. Todo o conteúdo da Revista está sob uma licença Creative Commons Atribuição-NãoComercial-Compartilhalgual 3.0 Não Adaptada. Ao serem publicados por esta Revista, os artigos são de livre uso em ambientes educacionais, de pesquisa e não comerciais, com atribuição de autoria obrigatória. Mais informações em http://www.atoz.ufpr.br/index.php/atoz/about/submissions\#copyrightNotice.

\begin{abstract}
Resumen
Introducción: Describe la utilización de las Redes Bayesianas para implementar un modelo de incertidumbre que permita predecir el estilo de aprendizaje de los estudiantes mediante la interacción en un entorno virtual de aprendizaje basado en el modelo de Felder-Silverman. Método: El modelo de incertidumbre se lo diseño y desarrolló para el funcionamiento en el LMS Moodle. Para validar el modelo propuesto se planteó un escenario educativo real conformado por dos grupos experimentales pertenecientes a la Universidad Nacional de Loja y Universidad Internacional del Ecuador.

Resultados: El bloque "Estilo de Aprendizaje" (EA) permitió a los estudiantes visualizar las probabilidades de cada dimensión de su EA observando que, de acuerdo a su interacción, cambiaban dichas probabilidades. De igual forma el docente pudo visualizar las probabilidades del EA que obtuvo cada estudiante al interactuar en un curso virtual alojado en el Entorno Virtual de Aprendizaje.

Conclusión: La propuesta podrá servir como apoyo al docente que desee identificar los estilos de aprendizaje predominantes de los estudiantes y, en base a ello, preparar actividades y recursos en su aula virtual.
\end{abstract}

Palavras-chave: Inteligencia artificial. Modelo bayesiano. Modelo Felder-Silverman.

\begin{abstract}
Introduction: It describes the use of Bayesian Networks to implement a model of uncertainty to predict the learning style of students through their interaction in a virtual learning environment based on the Felder-Silverman model.

Method: The model uncertainty was designed and developed to be integrated in the LMS Moodle. In order to validate the proposed model, an actual educational scenario was built and two groups - one from the National University of Loja and other from the International University of Ecuador - were exposed to the experiment.

Results: The block "Learning Style" (EA) allowed students to visualize the probabilities of each dimension of their EA by observing that, according to their interactions, these probabilities changed. Likewise, the teachers could visualize the probabilities of EA obtained by each student when these interactions were done in the hosted virtual course enclosed in the Virtual Learning Environment.

Conclusion: The proposal may serve as support for teachers who want to identify predominant learning styles of their students and, based on that, prepare activities and resources in the courses under their responsibilities.
\end{abstract}

Keywords: Artificial intelligence. Bayesian model. Felder-Silverman model.

\section{INTRODUCCIÓN}

En la actualidad se ha adquirido un gran interés en determinar cómo los estudiantes aprenden y adquieren el conocimiento en los Entornos Virtuales de Aprendizaje (EVA) (Cerrillo, 2004; Mestre, Fonseca, \& Valsés, 2007; Chía, \& Muñoz, [s.d.]; Mejía, 2009; Zapata-Ros, 2012; Veytia, 2013). Para cumplir con ello, sobre las plataformas virtuales se han diseñado y utilizado cuestionarios que permiten identificar el estilo de aprendizaje. Sin embargo, éste método ha demostrado no ser el adecuado debido que además de consumir tiempo, es un método poco fiable, puesto que los estudiantes tienden a elegir respuestas arbitrariamente siendo inconscientes de los usos futuros que se les puede dar a los resultados. Por lo tanto, la información obtenida puede ser inexacta y puede no reflejar los estilos de aprendizaje reales (Yannibelli, Godoy, \& Amand, 2006; González, 2009).

El concepto de estilos de aprendizaje (EA) surge en los diseños instruccionales de cursos virtuales que contienen información predeterminada referente al tema que se está abordando, pero muchas de las veces no es la adecuada, ni relevante y sobre todo innecesaria para cada estudiante. Esto se debe a que por medio del EVA no se aplica una evaluación previa, que sea confiable para detectar el EA y con ello poder conocer las necesidades de formación de cada estudiante, neutralizando así, las oportunidades de mejora en la enseñanza, como la desmotivación del estudiante para aprender de acuerdo a sus preferencias subjetivas.

Los EA se definen como la forma en la que las personas recopilan, procesan y organizan la información. Para su identificación existen instrumentos psicométricos útiles para averiguar el EA, tal es el caso del modelo de 
Felder-Silverman (FSLSM) ya que es uno de los modelos con mayor reputación y ha sido implementado con éxito en muchos sistemas de e-learning (Garcia, Amandi, Schiaffino, \& Campo, 2007; Carmona, Castillo, \& Millán, 2009; Sarango, 2012). El modelo de Felder-Silverman clasifica a los estudiantes en 4 dimensiones: procesamiento, percepción, entrada, comprensión; donde cada dimensión tiene un conjunto de estrategias que dirigen sus preferencias a ciertos recursos académicos tales como: videos, foros, chats, texto, imágenes, entre otros.

En el ámbito de la educación, particularmente en los EVA, las Redes Bayesianas (RB), conocidas como modelos probabilísticos, modelo bayesiano o red de creencia han sido objeto de investigación y de un creciente interés en cuanto a predecir los EA. Esto radica en que un modelo bayesiano está circunscrito, como técnica de pronóstico, cuya principal característica es la valoración o cualificación a hechos o datos observados. Su rol como instrumento de pronóstico es muy importante ya que permite hacer inferencias sobre la probabilidad de ocurrencia de una situación dada sobre la base de las evidencias observadas. Por ello, es un instrumento extraordinario para el monitoreo o seguimiento de situaciones de interés (Jesús, 2000).

Existen investigaciones que proponen a los modelos probabilísticos como una alternativa de solución innovadora en los entornos de educación virtual. Una investigación que se puede tomar de referencia es la Evaluación de RB (Garcia et al, 2007), siendo su objetivo el de utilizar la técnica para detectar el EA de acuerdo a los diferentes comportamientos que tiene el estudiante en el entorno virtual. Otra investigación, siendo relevante para el caso de estudio es el Modelo Bayesiano del Alumno basado en el EA y las Preferencias (Carmona et al, 2009), la misma que da a conocer un modelo de EA y un modelo de decisión para cada alumno, diseñado de acuerdo a las preferencias e interacciones del usuario con el sistema.

Bajo éste panorama, es importante que los EVA brinden información confiable acerca de la forma en que aprenden los estudiantes, siendo esto, información base para diseñar estrategias de enseñanza a fin de maximizar el proceso de aprendizaje en los entornos virtuales. Para este propósito, el presente trabajo da a conocer la implementación de un bloque basado en un modelo de red de creencia funcional para el LMS Moodle 2.5.4, el mismo que provee a los docentes, estudiantes y demás usuarios, un estimado de la probabilidad relacionada a cada dimensión de su EA, resultados que son generados de acuerdo a la interacción que mantiene el estudiante con los recursos y actividades disponibles en el EVA.

El articulo está estructurado de la siguiente manera: Introducción, presenta el objeto de estudio, estado del arte y trabajos relacionados; Metodología, detalla el modelo de la red bayesiana, su implementación y validación del modelo en el LMS Moodle; Resultados y discusión, presenta un análisis del experimento realizado, así como la discusión de los resultados obtenidos; Conclusiones, establece los logros alcanzados y las líneas futuras que se generó tras la culminación del trabajo.

\section{METODOLOGÍA}

Dentro de la metodología de la investigación se combinó el estudio de casos para la argumentación teórica del trabajo, la observación activa y el método de experimento que se utilizó para el control y seguimiento de las actividades de evaluación de la propuesta. Para poder implementar/validar la herramienta de apoyo al EVA que permita predecir para cada estudiante las probabilidades relacionadas a cada dimensión de su EA en base al modelo de Felder-Silverman se utilizó los procesos de la Ingeniería de Software diseñando una RB que fue implementada como un bloque "Estilo de Aprendizaje" para el LMS Moodle 2.5.4, que fue validado en un escenario educativo real mediante un grupo experimental conformado por dos grupos de estudiantes de la Universidad Nacional de Loja y Universidad Internacional del Ecuador.

Teniendo las bases teóricas fundamentadas y estudiadas (estudio de casos), la primera instancia fue modelar la red bayesiana, de acuerdo a los requerimientos obtenidos para la investigación (observación activa), luego se procedió a utilizar la metodología de desarrollo de software clásica (Ingeniería de Software) para el diseño e implementación del modelo probabilístico, descritas en las secciones siguientes. 


\section{Modelo de la Red Bayesiana}

El modelo de RB representa la relación existente entre las dimensiones del modelo del EA de Felder-Silverman y los factores que lo determinan, siendo estos la interacción del estudiante con los recursos y actividades disponibles en el EVA (Cuadro1):

a) recursos: material de aprendizaje (archivo, carpeta, página, libro);

b) actividades: chat, foro, cuestionario.

\begin{tabular}{|lc|}
\hline Chat & Procesamiento (Activo, Reflexivo ) Gráfico 1 \\
Foro & Escribe, lee mensajes, sin participación \\
Tiempo cuestionario & Participa foros, Lee foros, Sin participación \\
& Bajo, Normal, Alto \\
Visita material de aprendizaje & Percepción (Sensitivo, Intuitivo) Gráfico 2 \\
Calificación cuestionario & Visita, No visita. \\
Tiempo cuestionario & Bajo, Normal, Alto \\
& Bajo, Normal, Alto \\
Formato archivos material de aprendizaje & Entrada (Visual, Verbal) Gráfico 3 \\
Interacción foro & Visual (video, imágenes) \\
& Verbal (audio, texto) \\
Acceso material de aprendizaje & Interactúa, No interactúa. \\
Calificación cuestionario & Comprensión (Secuencial, Global) Gráfico 4 \\
\hline
\end{tabular}

Cuadro 1. Relación entre EA y recursos/actividades del EVA

Fuente: Elaboración propia.

En base a las variables definidas en el Cuadro 1, se diseñó la estructura de la RB para cada dimensión del EA, la misma que está conformada por los Nodos padres (Interacción EVA), siendo nodos independientes, y los Nodos hijos (Dimensión EA).

[1]

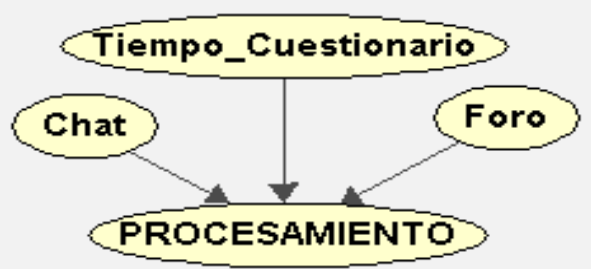

[3]

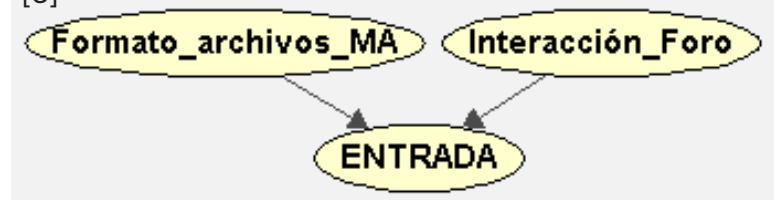

[2] Visita_MA

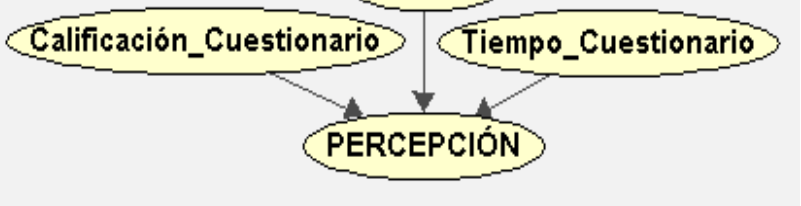

[4]

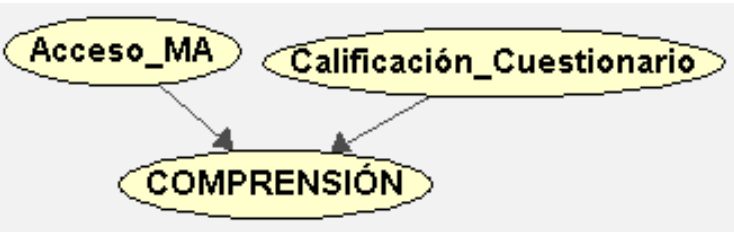

Figuras 1-4. [1] Dimensión procesamiento. [2] Dimensión percepción. [3] Dimensión entrada. [4] Dimensión comprensión. Fuente: Elaboración propia.

Las 4 RB (Figuras 1-4) en su conjunto integran un modelo de RB final como se observa en el figura 5, permitiendo con ello estimar las probabilidades en cada dimensión del EA.

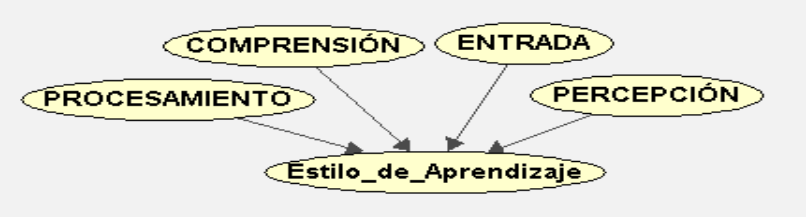

Figura 5. Red bayesiana final. Fuente: Elaboración propia. 
La predicción del EA en la RB final, se realiza mediante el proceso de inferencia, donde para ello es necesario definir las tablas de probabilidad asociadas a cada nodo. Por ello, la información útil para el proceso de inferencia está dada por:

a) la probabilidad a priori de los nodos padres: los valores para las tablas de probabilidad de los nodos Interacción EVA, se extrae de las evidencias que corresponde a la interacción que realiza el estudiante con el EVA;

b) la probabilidad condicionada de los nodos hijos: Para los nodos Dimensiones del EA, se determina una Tabla de Probabilidad Condicional (TCP), siendo esto base para realizar el proceso de inferencia en la RB. Se presenta a continuación el TCP del nodo comprensión (Tabla 1) y entrada (Tabla 2) siendo de referencia para los nodos hijos restantes. Los valores necesarios para la inferencia fueron estimados mediante datos recolectados y fuentes bibliográficas (Yu, Chen, 2006; Garcia et al., 2007; Graf, Kinshuk, \& Tzu-Chien, 2009).

\begin{tabular}{lc|c|c|c|c|c}
\hline \multirow{2}{*}{ Acceso al MA } & \multicolumn{3}{c}{ Continuo } & \multicolumn{3}{c}{ Saltos } \\
\cline { 2 - 7 } Calificación cuestionario & Bajo & Normal & Alto & Bajo & Normal & Alto \\
Secuencial & 0.60 & 0.80 & 1 & 0.40 & 0.20 & 0 \\
Global & 0.40 & 0.20 & 0 & 0.60 & 0.80 & 1 \\
\hline
\end{tabular}

Tabla 1. TCP Nodo Comprensión.

Fuente: Elaboración propia.

\begin{tabular}{lc|c|c|c}
\hline Formato Archivo MA & \multicolumn{2}{c}{ Visual } & Verbal \\
\cline { 2 - 5 } Interacción Foro & No Interactúa & Interactúa & 0.25 & Interactúa \\
Visual & 1 & 0.75 & 0.75 & 0 \\
Verbal & 0 & 0.25 & 1 & 1 \\
\hline
\end{tabular}

Tabla 2. TCP Nodo Entrada.

Fuente: Elaboración propia.

\section{Implementación del modelo en LMS Moodle}

El diseño de la RB se integró y codificó en un bloque llamado “Estilo de Aprendizaje” para el LMS Moodle versión 2.5.4.

En la figura 6 se observa el bloque cuya arquitectura final, altamente modular, contiene una estructura de directorios, siendo el directorio principal ea.

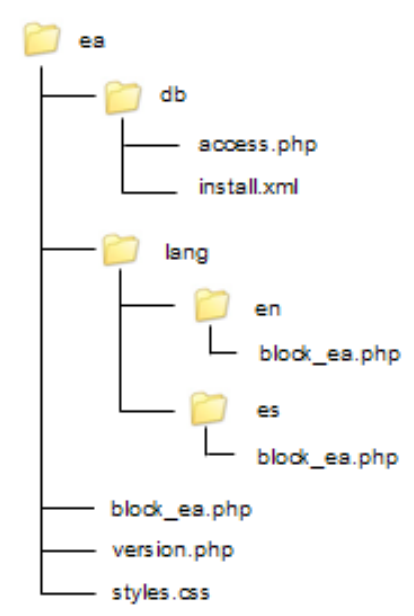

Figura 6. Directorio bloque ea.

Fuente: Elaboración propia.

El directorio ea contiene subdirectorios y ficheros que cumple con una funcionalidad específica, los mismos que se detallan a continuación:

a) $\mathrm{db}$ : directorio que contiene el fichero con los permisos particulares del bloque (access.php) y el fichero para crear las tablas del bloque EA en la base de datos Moodle (install.xml). Se crea una tabla 
para cada nodo Interacción EVA que almacena las evidencia para cada estudiante, y una tabla para cada nodo Dimisiones del EA, que almacena las probabilidades condiciones (TCP) útiles para el proceso de inferencia;

b) lang: directorio que contiene todos los ficheros de idioma, para ello se crea una carpeta y un fichero por cada idioma, en el caso del bloque EA está desarrollado para el idioma Inglés (en) y Español (es);

c) block_ea.php (archivo principal del bloque): fichero que integra el funcionamiento de la RB, así como funciones complementarias;

d) version.php: hace referencia a la versión del bloque;

e) styles.css: fichero que se usa para controlar la forma en que se ven los elementos visuales (diseño) que forman parte del bloque "Estilo de Aprendizaje".

\begin{tabular}{|c|c|}
\hline Método & Descripción \\
\hline init() & Método nativo de Moodle usado para inicializar el bloque. \\
\hline get_content() & $\begin{array}{l}\text { Método nativo de Moodle usado para mostrar el contenido del } \\
\text { bloque. El método genera la llamada a todos los métodos creados } \\
\text { por el desarrollador para su respectiva ejecución, y de esta forma } \\
\text { predecir el EA asociado a cada estudiante. } \\
\text { - cargar_tcp() } \\
\text { - } \quad \text { extraer_actualizar_evidencias(\$userid) } \\
\text { - inferenciaRB(\$userid) } \\
\text { - listarEstudiantes() } \\
\text { - } \quad \text { estrategias() }\end{array}$ \\
\hline cargar_tcp() & $\begin{array}{l}\text { Función que carga los datos que son base para el proceso de infer- } \\
\text { encia. El método carga en las tablas de los nodos Dimensión EA, } \\
\text { los datos definidas en las tablas de probabilidad condicional. }\end{array}$ \\
\hline $\begin{array}{l}\text { extraer_actualizar_evidencias } \\
\text { (\$userid) }\end{array}$ & $\begin{array}{l}\text { El método obtiene las evidencias asociadas a cada estudiante, sien- } \\
\text { do almacenadas en las tablas de los nodos Interacción EVA. }\end{array}$ \\
\hline $\begin{array}{l}\text { inferenciaRB } \\
\text { (\$userid) }\end{array}$ & $\begin{array}{l}\text { El método de acuerdo a la información almacenada en las tablas de } \\
\text { los nodos Interacción Eva y de los nodos Dimensión EA, procede a } \\
\text { realizar la inferencia utilizando como motor principal el Teorema de } \\
\text { Bayes. La inferencia se realiza para las cuatro dimensiones del EA: } \\
\text { - Procesamiento } \\
\text { - Percepción } \\
\text { - Comprensión } \\
\text { - Entrada } \\
\text { A más de ello, almacena en la tabla block_ea de la Base de Datos de } \\
\text { Moodle los resultados obtenidos de la inferencia. }\end{array}$ \\
\hline listarEstudiantes() & $\begin{array}{l}\text { Método creado para listar los estudiantes de un curso con su EA. } \\
\text { Este método es llamado cuando el usuario está en el curso con el } \\
\text { rol de administrador, profesor y profesor sin permisos de edición. }\end{array}$ \\
\hline estrategias() & $\begin{array}{l}\text { Método creado para mostrar información sobre como aprenden los } \\
\text { estudiantes según las } 4 \text { dimensiones del EA. }\end{array}$ \\
\hline
\end{tabular}

Cuadro 2. Métodos implementados bloque ea.

Fuente: Elaboración propia.

Los métodos mencionados en el Cuadro 2, relacionados al funcionamiento de la RB, se ejecutan cuando el usuario ingresa al entorno e interactúa con los recursos y actividades disponibles en el EVA.

\section{Validación del bloque estilo de aprendizaje}

Ya concluido el proceso de codificación de la RB, se procedió a implantar el bloque en el EVA basado en Moodle en el URL [http://www.estiloaprendizaje.com] a fin de monitorear y validar su funcionamiento. Para ello se estableció y creó un diseño instruccional de un curso virtual sobre "Introducción a Redes Bayesianas", a fin de que dos grupos experimentales conformado por estudiantes universitarios interactúen en el mismo y en base a ello observar el comportamiento del modelo de incertidumbre.

El bloque desarrollado para Moodle 2.5.4 genera como resultado para cada estudiante las probabilidades estimadas de su EA. El mismo que muestra la información dependiendo del rol que tiene asignado el usuario en el contexto del curso: 
a) cuando el estudiante no ingresa sus credenciales en la página principal del EVA o ingreso con el rol de invitado, el bloque "Estilo de Aprendizaje" muestra su portada principal (figura 7).

b) cuando el usuario tiene asignado el rol de administrador, creador de cursos, profesor o profesor sin permisos de edición, el bloque muestra la lista de los estudiantes que pertenecen al curso, con las probabilidades de las dimensiones de su EA (figura 8).

c) cuando el usuario tiene asignado el rol de estudiante, el bloque muestra las probabilidades que tiene en cada una de las dimensiones del EA (figura 9).
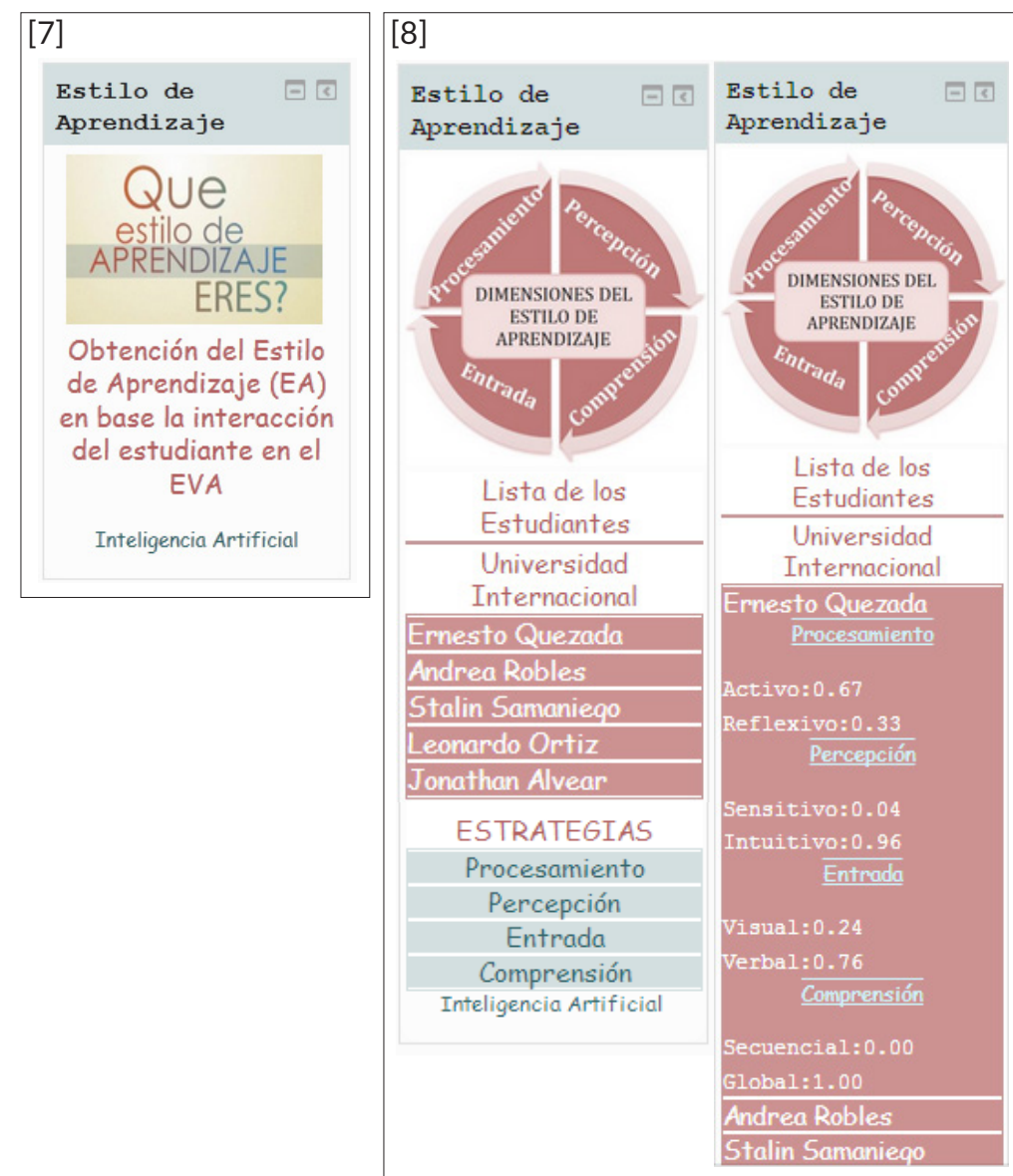

[9]

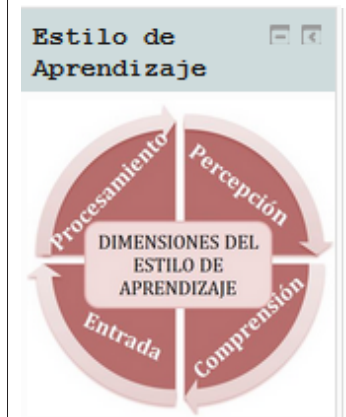

Porcentaje en las dimensiones de su Estilo de Aprendizaje

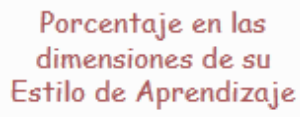

Procesamiento

Percepción

Entrada

Comprensión

ESTRATEGIAS

Procesamiento

Percepción

Entrada

Visual

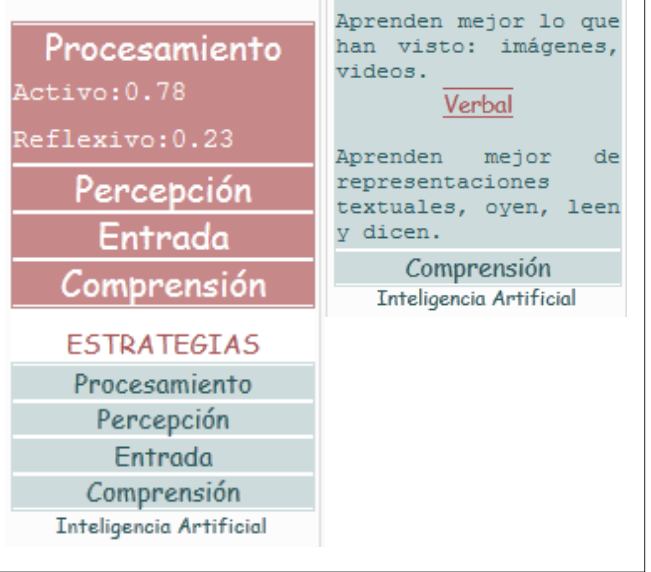

Figuras 7-9. [7] Bloque estilo de aprendizaje. [8] Bloque rol docente. [9] Bloque rol estudiante. Fuente: Elaboración propia.

Adicional, cuando el usuario se encuentra en el rol de estudiante, administrador, creador de cursos, profesor o profesor sin permisos de edición, el bloque da a conocer información referente al proceso de aprendizaje según las 4 dimensiones del EA.

\section{RESULTADOS Y DISCUSIÓN}

El bloque "Estilo de Aprendizaje" implementado en el EVA URL [http://www.estiloaprendizaje.com] luego de la experimentación realizada con los 2 grupos experimentales, permitió estimar para cada estudiante las probabilidades relacionadas a las 4 dimensiones del EA siendo estas: procesamiento, percepción, entrada, comprensión.

Para contrastar lo mencionado, fue necesario que en el EVA, se realice un diseño instruccional de un curso virtual sobre la temática de "Introducción a las Redes Bayesianas" siendo éste implementado en el EVA (Cuadro 3).

Cada sección del curso de RB estuvo compuesto por recursos y actividades de aprendizaje, las cuales de detallan en el Cuadro 4. 
La finalidad del diseño instruccional en el curso del EVA fue la de permitir que el grupo experimental conformado por estudiantes universitarios interactúen con los recursos y actividades disponibles en el curso y en base a ello el bloque "Estilos de Aprendizaje" genere resultados. La población del Grupo Experimental que interactuaron en este proceso correspondió a 27 estudiantes divididos en dos grupos, 22 estudiantes de décimo módulo paralelo "A" de la carrera de Ingeniería en Sistemas de la Universidad Nacional de Loja período marzo 2014 - julio 2014 y 5 estudiantes de la Universidad Internacional del Ecuador sede Loja (Cuadro 5). El curso virtual estuvo bajo la observación-dirección de un tutor/docente experto en contenidos de Inteligencia Artificial, teniendo una duración de 22 días de interacción en el curso, (lunes a viernes), con fecha de inicio: martes, 29 de abril del 2014 y fecha de culminación miércoles, 28 de mayo del 2014.

\begin{tabular}{|l|l|}
\hline \multicolumn{2}{|c|}{ Curso de Introducción a las Redes Bayesianas } \\
\hline \multicolumn{1}{|c|}{ Secciones } & \multicolumn{1}{c|}{ Descripción } \\
\hline Bloque: Bienvenida & $\begin{array}{l}\text { Dedicado a proporcionar } \\
\text { información del curso así como } \\
\text { a obtener las expectativas } \\
\text { que tiene los estudiantes del } \\
\text { mismo. }\end{array}$ \\
\hline $\begin{array}{l}\text { Sección 1: Introducción a las } \\
\text { Redes Bayesianas. }\end{array}$ & $\begin{array}{l}\text { Se enfoca a proporcionar los } \\
\text { recursos y actividades que } \\
\text { permitan a los estudiantes } \\
\text { adentrase a las RB conteniendo } \\
\text { los conceptos básicos para que } \\
\text { los estudiantes comprendan el } \\
\text { funcionamiento de la temática. }\end{array}$ \\
\hline Sección 2: Teorema de Bayes en & $\begin{array}{l}\text { Proporciona el material y } \\
\text { actividades útiles para que } \\
\text { el estudiante comprenda el } \\
\text { funcionamiento del Teorema de } \\
\text { Bayes en cuanto a realizar la } \\
\text { inferencia en la RB. }\end{array}$ \\
\hline $\begin{array}{l}\text { Sección 3: Algoritmos o Técnicas } \\
\text { para la Inferencia. }\end{array}$ & $\begin{array}{l}\text { Da a conocer diferentes algorit- } \\
\text { mos que son útiles para realizar } \\
\text { la inferencia en las RB. }\end{array}$ \\
\hline Sección 4: Herramientas. & $\begin{array}{l}\text { Tiene como objetivo dar a } \\
\text { conocer a los estudiantes algu- } \\
\text { nas herramientas que existen } \\
\text { para la aplicación de las RB. }\end{array}$ \\
\hline
\end{tabular}

Cuadro 3. Diseño instruccional. Fuente: Elaboración propia.

\begin{tabular}{|l|l|}
\hline \multicolumn{1}{|c|}{ Recursos } & \multicolumn{1}{|c|}{ Actividades } \\
\hline - $\begin{array}{l}\text { Materiales de lectura: } \\
\text { archivos en diferentes } \\
\text { formatos (docx, pdf, ppt, } \\
\text { jpeg, png, gif). }\end{array}$ & $\begin{array}{l}\text { Actividades individuales de } \\
\text { aplicación de lo estudiado tales } \\
\text { como. }\end{array}$ \\
- Materiales de lectura com- & $\begin{array}{l}\text { Foros de discusión. } \\
\text { plementaria (carpeta) } \\
\text { Libro en el formato del } \\
\text { EVA. } \\
\text { Página en el formato del } \\
\text { EVA. } \\
\text { Chat. }\end{array}$ \\
\hline
\end{tabular}

Cuadro 4. Recursos y actividades del curso. Fuente: Elaboración propia.

\begin{tabular}{|l|l|}
\hline \multicolumn{1}{|c|}{ Descripción } & \multicolumn{1}{c|}{ Usuarios } \\
\hline Universidad Nacional de Loja & 22 estudiantes \\
\hline $\begin{array}{l}\text { Universidad Internacional del } \\
\text { Ecuador }\end{array}$ & 5 estudiantes \\
\hline $\begin{array}{l}\text { Tutor/Docente de la Carrera de } \\
\text { Ingeniería en Sistemas. }\end{array}$ & Docente \\
\hline
\end{tabular}

Cuadro 5. Usuarios para el proceso de validación. Fuente: Elaboración propia.

El modelo de la RB implementado en el bloque "Estilo de Aprendizaje" para Moodle 2.5.4, generó resultados cuantitativos sobre los valores de los EA de todos los integrantes del grupo experimental observables luego de finalizar las temáticas del curso virtual alojado en el EVA.

El bloque "Estilo de Aprendizaje” permitió a los estudiantes visualizar las probabilidades de cada dimensión de su EA, observando que de acuerdo a su interacción cambiaban dichas probabilidades. De igual forma el docente pudo visualizar las probabilidades del EA que obtuvo cada estudiante al interactuar en el curso de “Introducción a las Redes Bayesianas".

Finamente el bloque, fue desarrollado para una función perfectamente definida, el mismo que recae sobre brindar información que sea de apoyo para mejorar el proceso enseñanza aprendizaje en los EVA. Esta información mostrada por el bloque, que son las probabilidades estimadas que tiene un estudiante en cada una de las dimensiones del EA, permite orientar a los usuarios a estar informados sobre el proceso de aprendizaje que se dan en los cursos virtuales mediados por EVA. Por otro lado, el docente al saber cómo los estudiantes aprenden constituye otra de las principales aportaciones del trabajo, ya que en base a la información suministrada por el bloque, el docente encargado de impartir el curso puede diseñar estrategias que permitan mejorar el proceso de enseñanza aprendizaje en la educación virtual. 


\section{CONCLUSIONES}

Las RB diseñan modelos que permitan llevar acabo predicciones. Por ello, por medio de las RB y sus algoritmos de inferencia, se pudo diseñar un modelo para ser implementado en el bloque "Estilo de Aprendizaje" para Moodle 2.5.4, generando así el diagnóstico de la forma en que aprenden los estudiantes, siendo éste el de predecir las probabilidades asociadas a cada dimensión del EA del modelo de Felder-Silverman.

La información generada por el bloque puede ser utilizada para diferentes propósitos. Uno de ellos sería el diseñar estrategias relacionado a los EA a fin de maximizar el aprovechamiento del aprendizaje en los entornos de educación virtual. También, es necesario mencionar que la modularidad del bloque desarrollado para el LMS Moodle deja abierta la puerta para que nuevos desarrolladores e investigadores del tema de la educación virtual mejoren con nuevos aspectos el modelo propuesto.

Los valores obtenidos en la predicción por medio de las RB, pueden servir a los docentes que trabajan sobre entornos virtuales de aprendizaje a mejorar sus diseños instruccionales y de esta manera proponer actividades y recursos de acuerdo al estilo de aprendizaje ya identificados.

La RB para predecir el EA en el EVA puede ser mejorada en diferentes aspectos, como por ejemplo: a) redefinir el modelo de la RB, identificando para ello nuevas variables relacionadas a los recursos y actividades que dispone un EVA (las mismas que deben ser útiles y relevantes para la inferencia en la red); b) efectuar una actualización de los valores definidas en las tablas de probabilidad condicional de los nodos dimensión EA, con el fin de acrecentar la validez del proceso de inferencia en la RB, garantizando con ello mayor confianza en la estimación de las probabilidades del EA.

Al tratarse de un bloque que brinda información sobre el EA del estudiante, se puede incorporar nuevas funcionalidades, siendo una de ellas, adaptar los contenidos del EVA de acuerdo a las características individuales que posee cada estudiante.

Se puede combinar el modelo bayesiano descrito en la investigación, con otras técnicas de Inteligencia Artificial como Procesamiento de Lenguaje Natural para identificar por medio los foros los estilos de aprendizaje al procesar los aportes-escritos que han hecho en sus diferentes interacciones en los temas que participen los estudiantes/tutores. 


\section{REFERÊNCIAS}

Cerrillo, Q. (2004). Aprendizaje Colaborativo y Redes de Conocimiento. Actas de las Jornadas Andaluzas de Organización y Dirección de Instituciones Educativas. Granada-España. Grupo Editorial Universitario, 9.

Chía, L., \& Muñoz, A. (n.d.). Adaptación de las plataformas e-learning a los estilos de aprendizaje utilizando sistemas multiagentes. Universidad Libre Cali. Colombia.

Carmona, C., \& Castillo, G., Millán, E. (2009). Modelo Bayesiano del alumno basado en el estilo de aprendizaje y las preferencias. IEEE-RITA, 4(2), 139-146. Retirado de http://rita.det.uvigo. es/200905/uploads/IEEE-RITA.2009.V4.N2.A8.pdf

González, H. (2009). Modelo dinámico del estudiante en cursos virtuales adaptativos utilizando técnicas de inteligencia artificial. (Tesis de Maestria - Magister en Ingeniería de Sistemas). Universidad Nacional de Colombia.

Garcia, P., Amandi, A., Schiaffino S., \& Campo, M. (2007). Evaluating Bayesian networks precision for detecting students learning styles. Computers \& Education, 49(3), 794-808. doi:10.1016/j. compedu.2005.11.017

Graf, S., Kinshuk, \& Liu, T-C. (2009). Supporting Teachers in Identifying Students Learning Styles in Learning Management Systems. Educational Technology \& Society, 12(4), 3-14. Retirado de http://www.ifets.info/journals/12_4/2.pdf

Jesús, E. A. (2000). Manual de metodologías, tomo II: La Técnica Bayesiana. Naciones Unidas para el Desarrollo Industrial, Programa de Prospectiva Tecnológica para Latinoamérica y el Caribe.
Mejía, C. (2009). Proceso de adaptación para entregar contenido basado en Estilos de aprendizaje del usuario. (Tesis de Maestria - Máster en Informática y Automática Industrial). Universidad de Girona.

Mestre, U., Fonseca, J., \& Valsés, R. (2007). Entornos virtuales de enseñanza aprendizaje. Editorial Universitaria.

Sarango, M. (2012). Aplicación de técnicas de minería de datos para identificar patrones de comportamientos relacionados con las acciones del estudiante con el EVA de. (Tesis de Grado - Ingeniero en Sistemas Informáticos y Computación). Universidad Técnica Particular de Loja.

Veytia, M. (2013). Cinco dimensiones para favorecer la apropiación tecnológica en estudiantes virtuales. Actas del Encuentro Internacional de Educación a Distancia. Universidad de Guadalajara, 21.

Yannibelli, V., Godoy, D., \& Amand, A. (2006). A genetic algorithm approach to recognize students' learning styles. Interactive Learning Environment, 14(1), 2006. doi:10.1080/10494820600733565

Yu, D., \& Chen, X. (2006). Using bayesian networks to implement adaptivity in mobile learning. Proceedings of the International Conference on Semantics, Knowledge and Grid, 2. doi:10.1109/ SKG.2006.107

Zapata-Ros, M. (2012). Teorías y modelos sobre el aprendizaje en entornos conectados y ubicuos: bases para un nuevo modelo teórico a partir de una visión crítica del "conectivismo". Universidad de Alcalá.

\section{Como citar este artigo (ABNT):}

LÓPEZ-FAICAN, L. G.; CHAMBA-ERAS, L. A. Redes bayesianas para predecir el estilo de aprendizaje de estudiantes en entornos virtuales. AtoZ: novas práticas em informação e conhecimento, Curitiba, v. 3, n. 2, p. 107-115, jul./dez. 2014. Disponível em: < http:// www.atoz.ufpr.br>. Acesso em:

\section{How to cite this article (APA):}

López-Faican, L. G., \& Chamba-Eras, L. A. (2014). Redes bayesianas para predecir el estilo de aprendizaje de estudiantes en entornos virtuales. AtoZ: novas práticas em informação e conhecimento, 3(2), 107-115. Retrieved from http://www.atoz.ufpr.br 